\title{
Ex vivo construction of rabbit corneal endothelial cell sheets on a porcine descemet membrane graft
}

\author{
CHUNYU LIU, SHENFEI TANG, GUOZHEN NIU, JUAN ZHANG, \\ XINYU HUANG, YUSHAN ZHANG and YANLONG BI \\ Department of Ophthalmology, Tongji Hospital Affiliated with Tongji University School of Medicine, \\ Shanghai 200333, P.R. China
}

Received July 30, 2018; Accepted March 26, 2019

DOI: $10.3892 / \mathrm{etm} .2019 .7573$

\begin{abstract}
The aim of the present study was to investigate the feasibility of a new graft construction method using rabbit corneal endothelial cells (RCECs) and a porcine descemet membrane (DM) carrier. RCECs were isolated and the experimental group was treated with Y-27632, whereas the control group were cultured in medium without Y-27632. RCEC morphology was observed using an inverted microscope, and cell proliferation and apoptosis were detected by flow cytometry. To confirm the presence of RCECs, reverse transcription-quantitative PCR was used to detect gene expression levels of $\mathrm{Na}+\mathrm{K}+\mathrm{-ATPase}$, aquaporin 1, collagen $\alpha_{2}$ (IV), collagen $\alpha_{1}$ (VIII) and keratin-12. Histocompatibility testing was used to detect porcine DM antigenicity. A DM-RCEC graft was constructed, and morphology was observed using alizarin red-trypan blue and haematoxylin and eosin staining. Cell membrane potential was measured to evaluate the physical function of the DM-RCEC graft. Complex graft tension was measured using a modified tension detector and compared with fresh porcine DM-endothelium complex. In vitro-cultured RCECs formed a monolayer with a polygon morphology and cobblestone-like arrangement. In vitro-cultured RCECs exhibited typical RCEC characteristics before and after transplantation. The proliferation rates of the experimental and control groups were 62.68 and $34.50 \%$, respectively $(\mathrm{P}<0.05)$; the apoptosis rates of the experimental and control groups were 8.99 and $35.68 \%$, respectively $(\mathrm{P}<0.05)$. There was no antigenicity observed with the porcine DM. The action potential amplitude of the experimental and control groups was over $-80 \mathrm{mV}$, reflecting normal RCEC physiological function. The tension measurements of the experimental and control groups were $20.0248 \pm 1.048$ and $20.5013 \pm 0.657 \mathrm{~g}$, respectively
\end{abstract}

Correspondence to: Professor Yanlong $\mathrm{Bi}$, Department of Ophthalmology, Tongji Hospital Affiliated with Tongji University School of Medicine, 389 Xincun Road, Shanghai 200333, P.R. China E-mail: biyanlong@tongji.edu.cn

Key words: rabbit corneal endothelial cells, carrier, porcine Descemet's membrane, Y-27632
$(\mathrm{P}>0.05)$. Taken together, the results of the present study demonstrated that Y-27632 enhanced RCEC proliferation. In addition, the findings revealed the successful ex vivo construction of a RCEC sheet on a porcine DM graft.

\section{Introduction}

In 2006, Melles et al (1) presented Descemet's membrane endothelial keratoplasty (DMEK), a technique, which requires that the DM-endothelium complex is fabricated prior to the operation. The postoperative anatomical structure of DMEK conforms to the physiological state of the cornea (1), however, a worldwide shortage of donor cornea has limited its application. In vitro corneal endothelial cell (CEC) culture is expected to solve this problem.

In 1979, Gospodarowicz et al (2) seeded in vitro-cultured bovine CECs onto decellularized bovine corneas, a study which inspired several fabricated implant studies, including the seeding of CECs onto gelatine film (3), amniotic membrane (4), gelatine hydrogel (5) and a thin corneal stromal plate (6). Despite these efforts, maintaining the normal morphology and density of CECs in vivo remains a problem.

The corneal endothelium originates from the neural crest and lines the innermost layer of the cornea (7). Normal CECs are a hexagonal monolayer of flat cells, which arrange in a cobblestone-like morphology that form a physical barrier between the aqueous humour and the corneal stroma (8). Normal human CECs (HCECs) do not proliferate in vivo, due to arrest at the $\mathrm{G}_{1}$ phase (9). HCEC injury is primarily repaired by the expansion and migration of nearby cells that fill the damaged area (10). Proliferation of functional HCECs is difficult to achieve using standard cell culture techniques (11). However, HCECs have been successfully cultured in vitro with epidermal growth factor, platelet-derived growth factor, bovine pituitary extract and foetal bovine serum (10). However, after multiple passages, HCEC proliferation decreases significantly and changes in cell morphology occur (11).

Rho-associated protein kinases (ROCKs) are involved in a variety of cellular activities, which include cell adhesion, proliferation, metabolism, apoptosis and cell cycle regulation (12). Y-27632 is a selective ROCK inhibitor, which can be used to inhibit the Rho signalling pathway (13). In the current study, Y-27632 was added to the culture medium to enhance 
the proliferation of functional in vitro-cultured rabbit CECs (RCECs). A heterologous implant was constructed using the in vitro-cultured RCECs and a porcine DM carrier. To prevent immunological rejection and transplant failure, an antigen-free technique was used to improve histocompatibility. To the best of our knowledge, the method used to construct this heterologous implant in the present study has not been previously reported.

\section{Materials and methods}

RCEC isolation and cell culture. A total of 3 New Zealand white rabbits (female, $\mathrm{n}=2$; male, $\mathrm{n}=1$; mean body weight, $2.5 \mathrm{~kg}$ ) were provided by the Experimental Animal Center of the Tongji University School of Medicine. Rabbits were maintained under controlled conditions (temperature, $22 \pm 2^{\circ} \mathrm{C}$; humidity, 55 $\pm 5 \%$; 12-h light/dark cycles) and were allowed free access to food and water. Rabbits were sacrificed by an injection of sodium pentobarbital solution (100 mg/kg; Bayer) in the ear vein and their eyeballs were removed. Cornea were dissected and placed on a petri dish, endothelium side up. The DM-endothelium complex was isolated from the cornea under an anatomic microscope, tissue was minced thoroughly and cells were detached following treatment with $0.25 \%$ trypsin for $5 \mathrm{~min}$. Isolated cells were collected and centrifuged at $252 \mathrm{x} \mathrm{g}$ for $5 \mathrm{~min}$ at room temperature. The supernatant was removed and RCECs were cultured in Dulbecco's modified Eagle medium (DMEM)/F12 medium (HyClone; GE Healthcare Life Sciences) supplemented with 10\% FBS (HyClone; GE Healthcare Life Sciences), $1 \%$ penicillin-streptomycin solution (Beyotime Institute of Biotechnology) and 0.1\% Y-27632 (10 $\mu \mathrm{M}$; Sigma-Aldrich; Merck KGaA) (14), and maintained at $37^{\circ} \mathrm{C}$ in a $5 \% \mathrm{CO}_{2}$-humidified incubator. The culture medium was changed every 2 days. RCECs in the control group were cultured in DMEM/F12 without Y-27632. RCECs were passaged when cells reached $80-90 \%$ confluence. The current study was approved by the Ethics Committee of Tongji Hospital Affiliated with Tongji University School of Medicine (permit no. 42501068531010711A1001).

Flow cytometry. Logarithmic growth phase cells (80,000 cells) were isolated and centrifuged at $252 \mathrm{x} \mathrm{g}$ for $5 \mathrm{~min}$ at $37^{\circ} \mathrm{C}$. Cells were resuspended in $195 \mathrm{ml}$ of Annexin V-FITC binding buffer (Beyotime Institute of Biotechnology) and subsequently stained with $2 \mathrm{ml}$ of Annexin V-FITC. The solution was mixed and incubated in the dark for $10 \mathrm{~min}$ at room temperature. The cell suspension was centrifuged at $252 \mathrm{x} \mathrm{g}$ for $5 \mathrm{~min}$ at $37^{\circ} \mathrm{C}$, the supernatant was removed and cells were resuspended in $190 \mathrm{ml}$ of Annexin V-FITC binding buffer. Cells were subsequently incubated with $10 \mathrm{ml}$ of propidium iodide and incubated in the dark using an ice bath method, as previously described (15). Cells were filtered through a nylon mesh prior to analysis. Stained cell suspensions were detected using an AccuriC6 flow cytometer (BD Biosciences) and analyzed using FlowJo software (version 7.6.1; Tree Star, Inc.).

Reverse transcription-quantitative PCR (RT-qPCR). Total RNA was extracted from RCECs using TRIzol reagent (Invitrogen; Thermo Fisher Scientific, Inc.). Total RNA was reverse transcribed into cDNA using ExScript RT reagent kit (Invitrogen; Thermo Fisher Scientific, Inc.). qPCR was subsequently performed using SYBR Green PCR Master mix (Invitrogen; Thermo Fisher Scientific, Inc.), with a final volume of $20 \mathrm{ml}$ that included $10 \mu \mathrm{l} \mathrm{SYBR-green} \mathrm{mix,} 0.4 \mu \mathrm{l}$ 50X ROX reference Dye II, $2.5 \mu \mathrm{l}$ reverse primer $(1 \mu \mathrm{M}), 2.5 \mu \mathrm{l}$ forward primer $(1 \mu \mathrm{M}), 1 \mathrm{ml}$ diluted cDNA $(10 \mathrm{ng} / \mathrm{ml})$ and $3.6 \mathrm{ml}$ of $\mathrm{ddH}_{2} \mathrm{O}$. The target gene primer sequences were designed with PrimerPremier5 software (Premier Biosoft) and aligned in BLAST. The primers used are summarised in Table I. The following thermocycling conditions were used for qPCR: Initial denaturation at $95^{\circ} \mathrm{C}$ for $10 \mathrm{~min}$; followed by 39 cycles of $98^{\circ} \mathrm{C}$ for $30 \mathrm{sec}, 58^{\circ} \mathrm{C}$ for $30 \mathrm{sec}$ and $74^{\circ} \mathrm{C}$ for $30 \mathrm{sec}$. Relative mRNA expression levels were quantified using the $2^{-\Delta \Delta \mathrm{Cq}}$ method (16) and normalized to the internal reference gene GAPDH.

Immunocytochemistry. Third-generation CECs were seeded onto six-well plates at a density of $1 \times 10^{3}$ cells/well and cultured for up to 4 days. CECs were fixed in $95 \%$ ethanol for $15 \mathrm{~min}$ at room temperature. Cells were incubated with $0.3 \% \mathrm{H}_{2} \mathrm{O}_{2}$ for $10 \mathrm{~min}$ at $37^{\circ} \mathrm{C}$ to inhibit endogenous peroxidase activity. Subsequently cells were incubated with $2 \%$ goat serum albumin (Invitrogen; Thermo Fisher Scientific, Inc.) for $10 \mathrm{~min}$ at $37^{\circ} \mathrm{C}$. Cells were incubated with primary antibody directed against connexin (Cx)43 (1:400; cat. no. 13-8300; Invitrogen; Thermo Fisher Scientific, Inc.) overnight at $4^{\circ} \mathrm{C}$. Following primary incubation, cells were incubated with biotin-labeled goat anti-rabbit secondary antibodies (1:400; cat. no. 65-6120; Invitrogen; Thermo Fisher Scientific, Inc.) for $10 \mathrm{~min}$ at room temperature. After incubating with catalase-labeled streptomyces antibiotic proteins for $10 \mathrm{~min}$, cells were subsequently stained with 3,3'-diaminobenzidine (Sigma-Aldrich; Merck $\mathrm{KGaA}$ ) and observed under a phase-contrast microscope (magnification, $\mathrm{x} 40$ ). In the control group, cells were incubated with PBS instead of primary antibody.

Immunofluorescence. Third-generation CECs were seeded onto six-well plates at a density of $1 \times 10^{3}$ cells/well and cultured for up to 4 days. CECs were fixed in $4 \%$ formaldehyde for $10 \mathrm{~min}$ at room temperature. Cells were incubated with $0.2 \%$ Triton $\mathrm{X}-100$ for membrane permeabilization, prior to being blocked with $2 \%$ goat serum albumin for $30 \mathrm{~min}$ at room temperature. Cells were incubated with primary antibody directed against Cx43 (1:400) overnight at $4^{\circ} \mathrm{C}$. Cells were washed three times in PBS. Following this, cells were incubated with FITC goat anti-rabbit IgG secondary antibody (1:100; cat. no. 65-6120; Invitrogen; Thermo Fisher Scientific, Inc.) in the dark for $1.5 \mathrm{~h}$ at $37^{\circ} \mathrm{C}$. Cell nuclei were counterstained with DAPI for $5 \mathrm{~min}$ at room temperature and fluorescence was observed under a fluorescence microscope (magnification, $\mathrm{x} 40$ ). In the control group, cells were incubated with PBS instead of primary antibody.

Preparation of porcine DM as a carrier. A total of 3 pigs (; female, $\mathrm{n}=2$; male, $\mathrm{n}=1$; mean body weight, $280 \mathrm{~kg}$ ) were provided by Shanghai Qinnong Animal Husbandry Technology Co., Ltd. (license no. 310230000455889). Pigs were maintained under controlled conditions (temperature, $22 \pm 2{ }^{\circ} \mathrm{C}$; humidity, $55 \pm 5 \%$; 12-h light/dark cycles) and were allowed free access to food and water. Pigs were sacrificed by an injection of sodium pentobarbital solution $(100 \mathrm{mg} / \mathrm{kg})$ in the ear vein and their eyeballs were removed. Porcine corneas were dissected and placed on a petri dish, endothelium side up. A drop of Trypan 
Table I. Quantitative PCR primer sequences.

\begin{tabular}{llc}
\hline Gene & \multicolumn{1}{c}{ Primer sequence (5'-3') } & Product length (bp) \\
\hline $\mathrm{Na}^{+}$-K ${ }^{+}$-ATPase & F: TCTGTAACAGGGCGGTATT & 176 \\
GAPDH & R: GGTGGAGTTGAAGGGTATCT & 217 \\
& F: CAGTCTTCTGGGTGGCAGTG & \\
Collagen type IV $\alpha 2$ & R: AGCCAAACGGGTCATCATCTC & 137 \\
Collagen type VIII $\alpha 1$ & F: GGCTGGCGGAGTCTGTGGAT \\
& R: ACTCGATGAAGGGCGTGGC \\
AQP1 & F: GCAGACGGGCATCTTCAC \\
& R: CATCACGGGCTCGTTGTT \\
Keratin-12 & F: GCTCACCCACAACTTCAACAA & 172 \\
& R: ATCGCCGTCCAGGTCATAC \\
VDAC2 & F: TCTCCAAACCGCAGACAC \\
& R: GACCACTTCGCCATTCAC \\
VDAC3 & F: GAGAAAGUAUGUGAAGAUUTT \\
\hline
\end{tabular}

AQP1, aquaporin 1; F, forward; R, reverse.

A

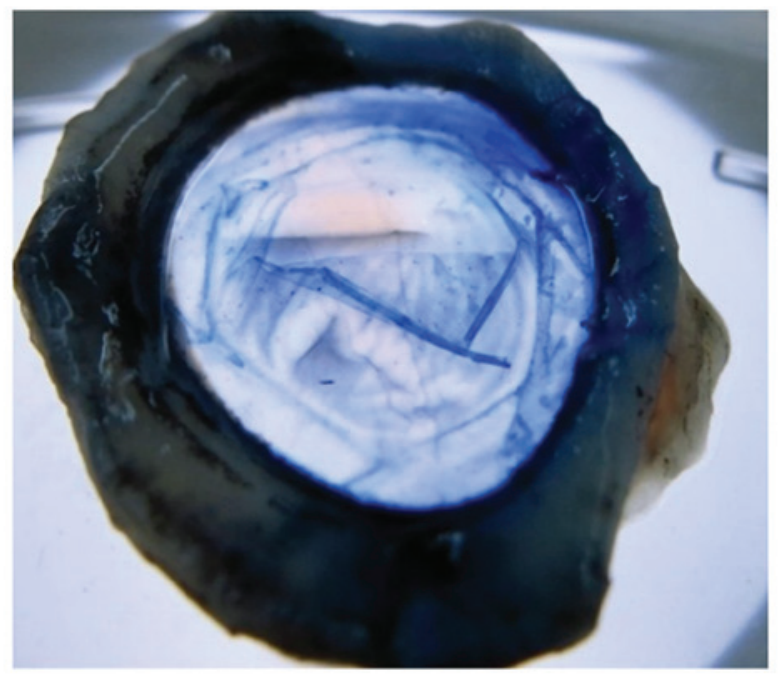

B

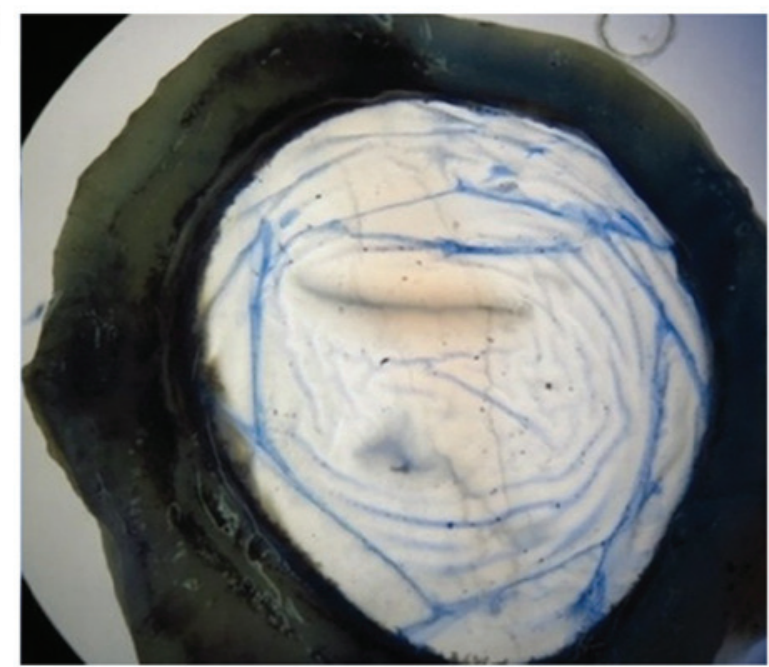

Figure 1. Isolation of the Descemet's membrane-endothelium complex. (A) The scalpel was used to lightly scratch the edge of the endothelium and (B) slowly tear the porcine Descemet's membrane-endothelial cell complex along the scratch edge.

blue solution (Sigma-Aldrich; Merck KGaA) was added to each cornea. The DM-endothelium complexes were isolated, and a $9 \mathrm{~mm}$ trephine was used to make a circular shape (Fig. 1). CECs were subsequently removed by gently wiping with a cotton swab under a microscope. After washing with $0.9 \% \mathrm{NaCl}$, the porcine DM carriers were preserved in liquid nitrogen for $48 \mathrm{~h}$, followed by storage in sterile glycerol at $4^{\circ} \mathrm{C}$ for three months.

Porcine DM antigenicity. A total of 6 wild-type female Swiss albino mice (age, 6-8 weeks; weight, 22 g) were provided by the Experimental Animal Center of the Tongji University School of Medicine (Shanghai, China). Mice were maintained under controlled conditions (temperature, $20-23^{\circ} \mathrm{C}$; humidity,
40-60\%; 12-h light/dark cycles) and were given access to water and food ad libitum. The porcine DM carriers were washed three times with PBS. The porcine DM carriers were implanted into mice enterocoelia $(n=6)$ and rabbit paravertebral muscle $(n=4)$, in rabbits following CEC isolation, after general anaesthesia with sodium pentobarbital (50 and $30 \mathrm{mg} / \mathrm{kg}$, respectively). In the control groups, mice enterocoelia $(n=3)$ and the rabbit contralateral muscle $(n=2)$ were incised without porcine DM carrier implantation. Close postoperative observation was performed to monitor vital signs, postoperative eating and activity behaviour. After 2 weeks, mice enterocoelia and rabbit paravertebral muscle was incised and observed. To examine the effect of porcine DM carrier implantation on 

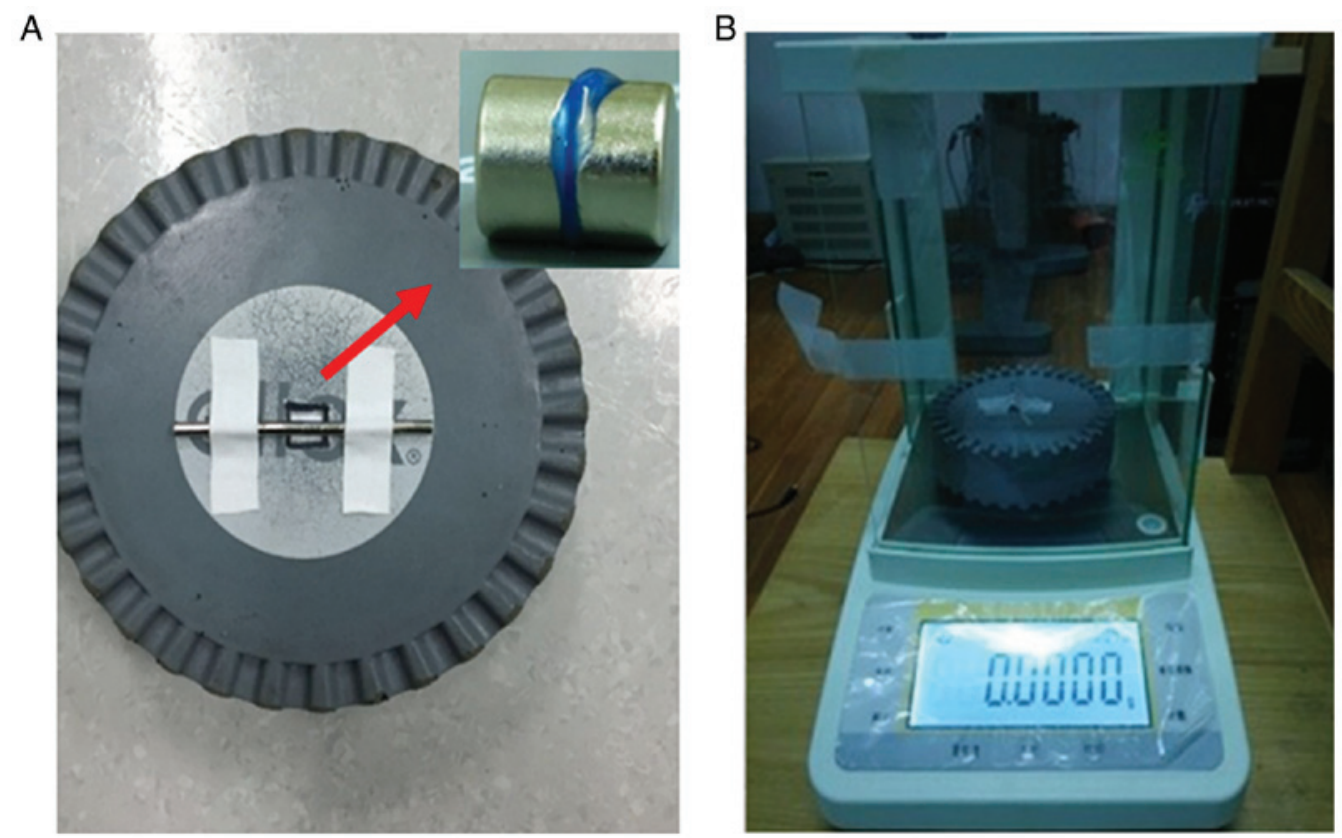

Figure 2. Modified tension detector. (A) An electronic balance was used to generate a modified tension detector, with foam padding used to isolate the magnetic field. (B) Each sample was flattened between two magnets, with adhesive tape used to immobilise the magnets.

splanchnic tissue and muscle, haematoxylin and eosin (H\&E) staining was performed, as previously described (17). Briefly, tissue samples were fixed in $10 \%$ formalin overnight at $4^{\circ} \mathrm{C}$ and embedded in paraffin. Paraffin-embedded tissue samples were cut into $3-\mu \mathrm{m}$-thick sections. Tissue sections were subsequently deparaffinized and rehydrated. Deparaffinized sections were stained with hematoxylin-imidine Red (HE) at room temperature for $3 \mathrm{~min}$. Any pathological changes were observed under a light microscope (magnification, $\mathrm{x} 40$ ).

Preparation of the porcine DM-RCEC complex. After the glycerol was removed, the porcine DM carriers were washed three times with $50 \mathrm{mg} / \mathrm{ml}$ vitriolic gentamicin (Bayer) and PBS buffer. RCECs that had been passaged twice in vitro were resuspended $\left(1 \times 10^{6}\right.$ cells $\left./ \mathrm{ml}\right)$. The porcine DM carriers $(\mathrm{n}=8)$ were placed in a six-well plate and the RCECs were seeded on top of the porcine DM carriers. The DM-RCEC mixture was cultured in DMEM/F12 at $37^{\circ} \mathrm{C}$ in a $5 \% \mathrm{CO}_{2}$-humidified incubator. Once cell adherence was observed, more culture medium was added to the plate. The complex was incubated until cell density reached $2,000-2,500$ cells $/ \mathrm{mm}^{2}$. The culture medium was changed once every 3 days.

Alizarin red-trypan blue staining. The porcine DM-RCEC complexes $(n=2)$ were transferred onto a glass slide with the endothelium side up. Cells were stained with $0.25 \%$ Trypan blue (Sigma-Aldrich; Merck KGaA) for $90 \mathrm{sec}$ at room temperature. Cells were washed with PBS and excess liquid was removed using filter paper. Cells were subsequently stained with $0.2 \%$ alizarin red $(\mathrm{pH} 4.2$; Sigma-Aldrich; Merck KGaA) for $90 \mathrm{sec}$ and rinsed twice with saline. The porcine DM-RCEC complexes were fixed with $2 \%$ glutaraldehyde (Beyotime Institute of Biotechnology) for $10 \mathrm{~min}$ at room temperature and observed under a microscope (magnification, $\mathrm{x} 40$ ).
Cell membrane potential measurement. RCECs obtained from the porcine DM-RCEC complexes were used as the experimental group $(n=4)$, whereas RCECs from fresh rabbit eyeballs were used as the control group $(n=4)$. A total of 4 New Zealand white rabbits (female, $n=2$; male, $n=2$; mean body weight, $2.5 \mathrm{~kg}$ ) were provided by the Experimental Animal Center of the Tongji University School of Medicine. Rabbits were maintained under controlled conditions (temperature, $22 \pm 2^{\circ} \mathrm{C}$; humidity, 55 $\pm 5 \%$; 12-h light/dark cycles) and were allowed free access to food and water. Rabbits were sacrificed by an injection of sodium pentobarbital solution $(100 \mathrm{mg} / \mathrm{kg}$; Bayer) in the ear vein and their eyeballs were removed. RCECs in both groups were prepared as a cell suspension $\left(1 \times 10^{6} \mathrm{cells} / \mathrm{ml}\right)$, transferred onto a glass slide and placed in a recording bath. Measurements were made in well-differentiated cells, which were observed using an immersion objective lens in the perfusate. A tight-seal, whole-cell recording patch-clamp technique was used to record the membrane potential (18). Briefly, the patch-clamp amplifier in voltage-clamp mode was used to seal the connection, while the microelectrode was used to generate a high-resistance up to $1 \mathrm{GW}$. After generating resistance, action potentials were recorded once the patch-clamp amplifier was in current-clamp mode. Data were analysed using PCLAMP 6.0 software (Molecular Devices, LLC).

Tension detection. RCECs obtained from the porcine DM-RCEC complexes were used as the experimental group $(\mathrm{n}=2)$, whereas fresh porcine DM-endothelium complex were used as the control group $(\mathrm{n}=2)$. Both groups comprised 10 circular samples, each $9 \mathrm{~mm}$ in diameter. An electronic balance was preheated for $30 \mathrm{~min}$ and circular foam padding was used to isolate the magnetic field (Fig. 2A). Each sample was flattened between two circular magnets $(8 \times 5 \mathrm{~mm})$, which were immobilized at the centre of the foam padding (Fig. 2B). 


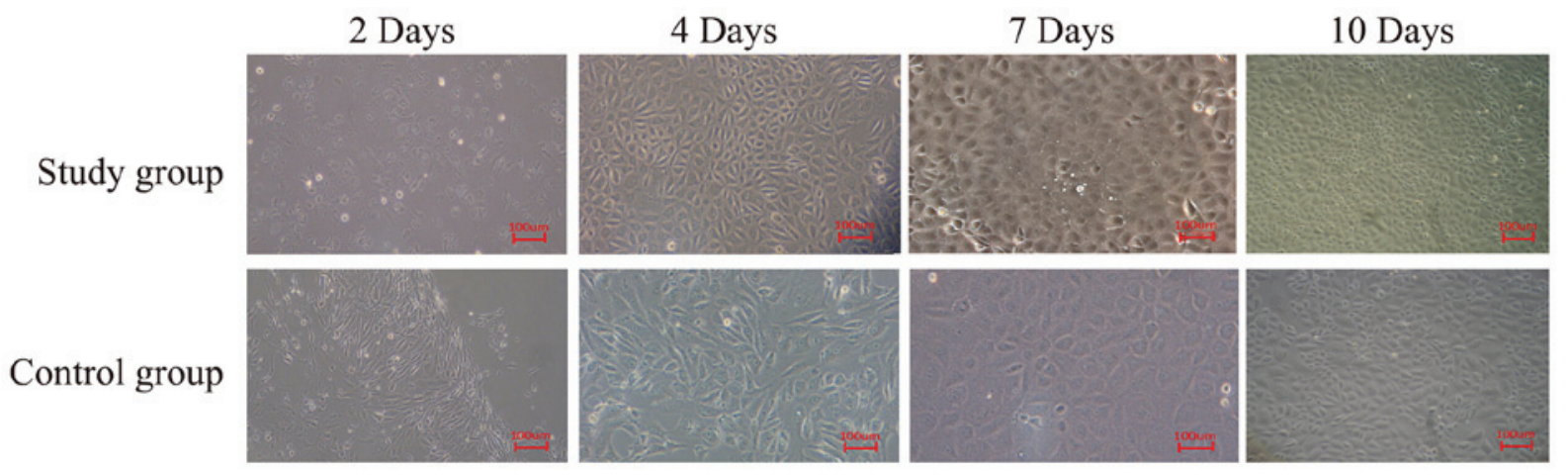

Figure 3. Rabbit corneal endothelial cell morphology. Cell morphology was observed in rabbit corneal endothelial cells in the experimental and control groups following culture for 2, 4, 7 and 10 days.

After peeling, the sample was strongly pulled in a vertical direction using antimagnetic microforceps. The value on the electronic balance was recorded when the sample broke, and the absolute value was taken as the sample's tension value.

Statistical analysis. Data presented as the mean \pm standard deviation. All statistical analyses were performed using SPSS software (version 11.0; SPSS, Inc.). Group means were analyzed using the $\chi^{2}$ test and Student's t-test. $\mathrm{P}<0.05$ was considered to indicate a statistically significant difference.

\section{Results}

RCEC cultures. Primary cultured RCECs were cultivated in a monolayer and cells displayed polygonal and cobblestone-like morphology. In addition, cells demonstrated strong proliferation ability and reached $80-90 \%$ confluence within 2 weeks. Following 24-h culture, there were more adherent RCECs in the experimental group compared with the control group (data not shown). At day 4, confluence occurred and cells demonstrated logarithmic growth. At day 7, cells demonstrated confluence and regular cellular morphology. At day 10, the second passage of RCECs was uniformly distributed and maintained cobblestone-like morphology. In addition, RCECs in the experimental group had reached $70 \%$ confluence compared with the control group, which had reached $55 \%$ confluence (Fig. 3). The second passage was established at day 12 and day 15 in the experimental and control groups, respectively (data not shown). RCECs gradually changed in morphology, with enlarged cell bodies in subsequent passages. At similar passage states, the RCECs in the experimental group had fewer morphological changes, fewer cytoplasmic bubbles and particles, and more active proliferation compared with the control group (data not shown). Based on these results, the second passage of RCECs from the experimental group was used to prepare the implant.

RCEC growth and apoptosis. The ratio of normal cells to total cells in the experimental and control groups were 86.64 and $59.30 \%$, respectively (Fig. 4A and B). The cell apoptosis rates of the two groups were 8.99 and $35.68 \%$, respectively $(\mathrm{P}<0.05$; Fig. 4A and $\mathrm{B})$. The R2/R1 value, an indicator of the RCEC proliferation rate, was significantly increased in the experimental group compared with the control group.
Table II. Ct and $2^{-\Delta \Delta \mathrm{Cq}}$ values of endothelial-associated markers.

\begin{tabular}{lccc}
\hline Gene & Average $\mathrm{Ct}$ & $\mathrm{SD}$ & $2^{-\Delta \Delta \mathrm{Cq}}$ \\
\hline GAPDH & 18.555 & 0.142606 & \\
$\mathrm{Na}^{+}-\mathrm{K}^{+}$-ATPase & 26.82833 & 0.740632 & 0.003 \\
Collagen $\alpha$ (IV) & 24.21967 & 0.052539 & 0.018 \\
Collagen $\alpha$ 1(VIII) & 24.90067 & 0.325694 & 0.011 \\
AQP1 & 22.25867 & 0.143420 & 0.068 \\
Keratin-12 & 30.6200 & 0.132556 & 0.000
\end{tabular}

$\mathrm{Ct}$, cycle threshold; SD, standard deviation; AQP1, aquaporin 1.

Confirmation of primary RCECs. The solubility-curve analysis was unimodal and demonstrated the specificity of the result (data not shown). The high cycle threshold $(\mathrm{Ct})$ values $(>30)$ and $2^{-\Delta \Delta C q}$ values $(=0)$ indicated low amounts of target sequence. As shown in Table II, the $\mathrm{Ct}$ and $2^{-\Delta \Delta \mathrm{Cq}}$ values of the epithelial-associated markers, $\mathrm{Na}^{+}-\mathrm{K}^{+}$-ATPase, collagen $\alpha 2$ (IV), collagen $\alpha 1$ (VIII), aquaporin (AQP)1 and keratin-12 indicated that the primary RCECs were endothelial cells. Immunostaining demonstrated that $\mathrm{Cx} 43$ expression was well preserved in the cytoplasm and cell membrane of in vitro CECs compared with the control (Fig. 5). The labeling rate of positively stained CECs was $~ 95 \%$, which suggests that CECs have increased activity and high expression levels of intercellular junction protein $\mathrm{Cx} 43$ in vitro (data not shown). These results suggest that the signal transduction, substance exchange and cell metabolism may be enhanced between CECs.

Porcine DM carrier antigenicity. During the 2-week porcine DM antigenicity test, mice and rabbits maintained normal activity, without demonstrating any psychological or limb disorders and no mortality was observed. All incisions healed well without infection or transudation. H\&E staining was performed two weeks following implanation. H\&E staining revealed that following implantation, normal cardiomyocyte, hepatocyte and nephrocyte morphology without any obvious abnormalities following implantation of the porcine DM carrier in mice enterocoelia in both the experimental and control groups (Fig. 6). In addition, H\&E staining demonstrated myofiber with clear microstructure and without signs 
A

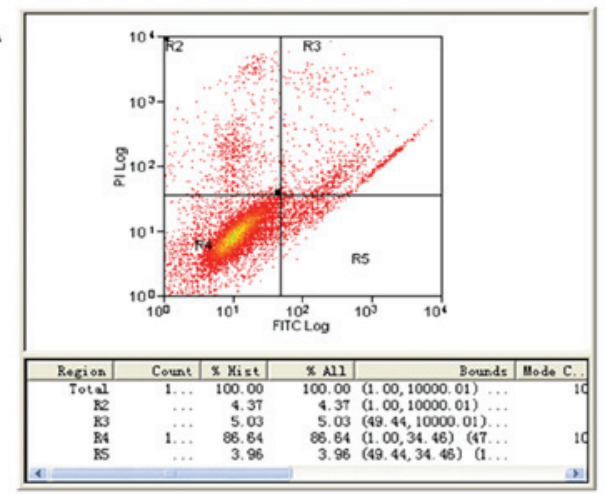

C

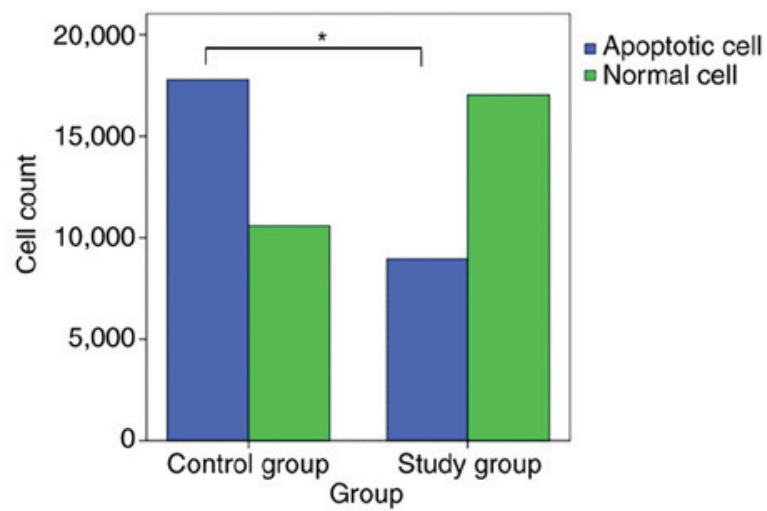

B

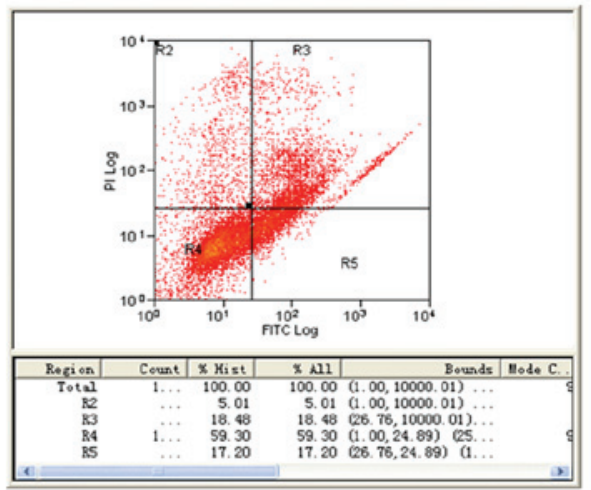

D

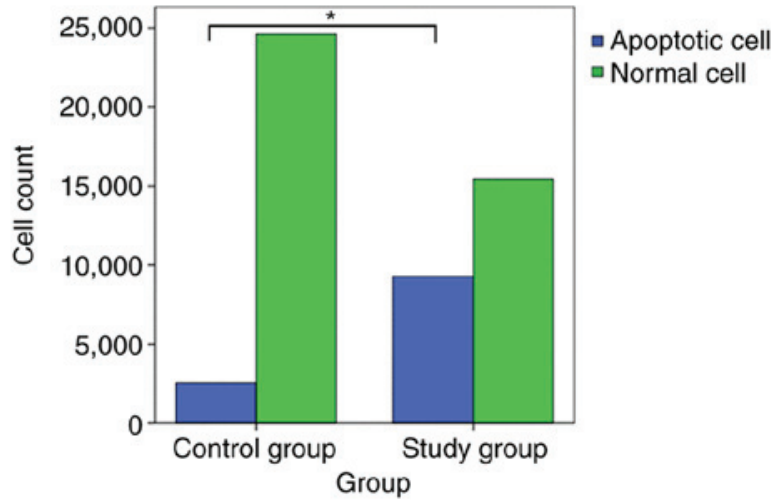

Figure 4. Cell proliferation and apoptosis. Representative flow cytometric analyses of the (A) experimental group and (B) control groups following Annexin V-FITC/PI staining. Events detected in the unstained quadrant (R4) represent normal cells, events detected in the FITC quadrant (R5) represent early apoptotic cells, events detected in the double-stained quadrant (R3) represent late apoptotic cells and the upper quadrant (R2) represents acceptable detection error. R3 and R5 quadrants represent all apoptotic cells. rabbit corneal endothelial cell (C) proliferation and (D) apoptosis rate were determined by Annexin V-FITC/PI staining and flow cytometry. $\mathrm{P}<0.05$ vs. control group. PI, propidium iodide.
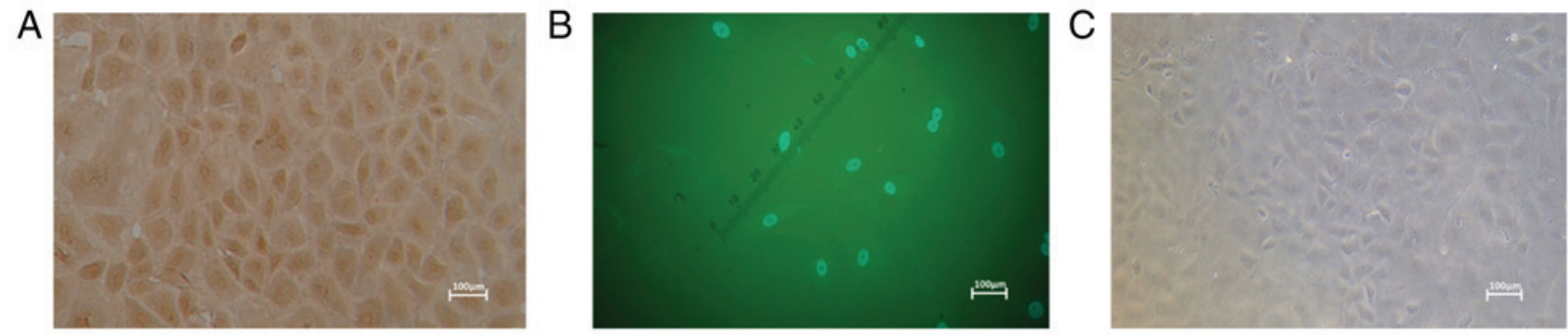

Figure 5. Immunostaining of RCECs. (A) Positive 3,3'-diaminobenzidine staining in CECs with dark brown cell nucleus and light brown cytoplasm and cell membrane. (B) Positive FITC staining in CECs demonstrated light yellow-green fluorescence in the cytoplasm and cell membrane. (C) No immunostaining in the control group. RCEC, rabbit corneal endothelial cell.

of inflammation, necrosis or degeneration in rabbit paravertebral muscle following implantation of the porcine DM carrier in the experimental and control groups (Fig. 7).

Confirmation of successful implantation. Alizarin red-trypan blue demonstrated that implanted RCECs adhered to the endothelium side of the porcine DM as a monolayer, which were tightly connected with a cell density of $2,000-2,500 / \mathrm{mm}^{2}$ and cells exhibited hexagonal and cobblestone-like morphology (Fig. 8A). H\&E staining following implantation revealed that in the experimental group, RCECs adhered to the surface of porcine DM uniformly (Fig. 8B), similar to that observed in the control group of fresh RCEC-DM complexes (Fig. 8C). The expression levels of voltage-dependent anion-selective channel-2 (VDAC2), voltage-dependent anion-selective channel-3 (VDAC3) and AQP1 in implanted RCECs were determined by RT-qPCR. The positive expression of VDAC2, VDAC3 and AQP1 in implanted RCECs correspond with typical RCEC gene expression characteristics (Fig. 8D). The action potential amplitudes of the implant RCECs (experimental group) and the freshly isolated RCECs (control group) were over $-80 \mathrm{mV}$ (Fig. $8 \mathrm{E}$ and F), and the experimental group had a significantly higher amplitude of the action potential compared with the control group $(\mathrm{P}<0.05$; 

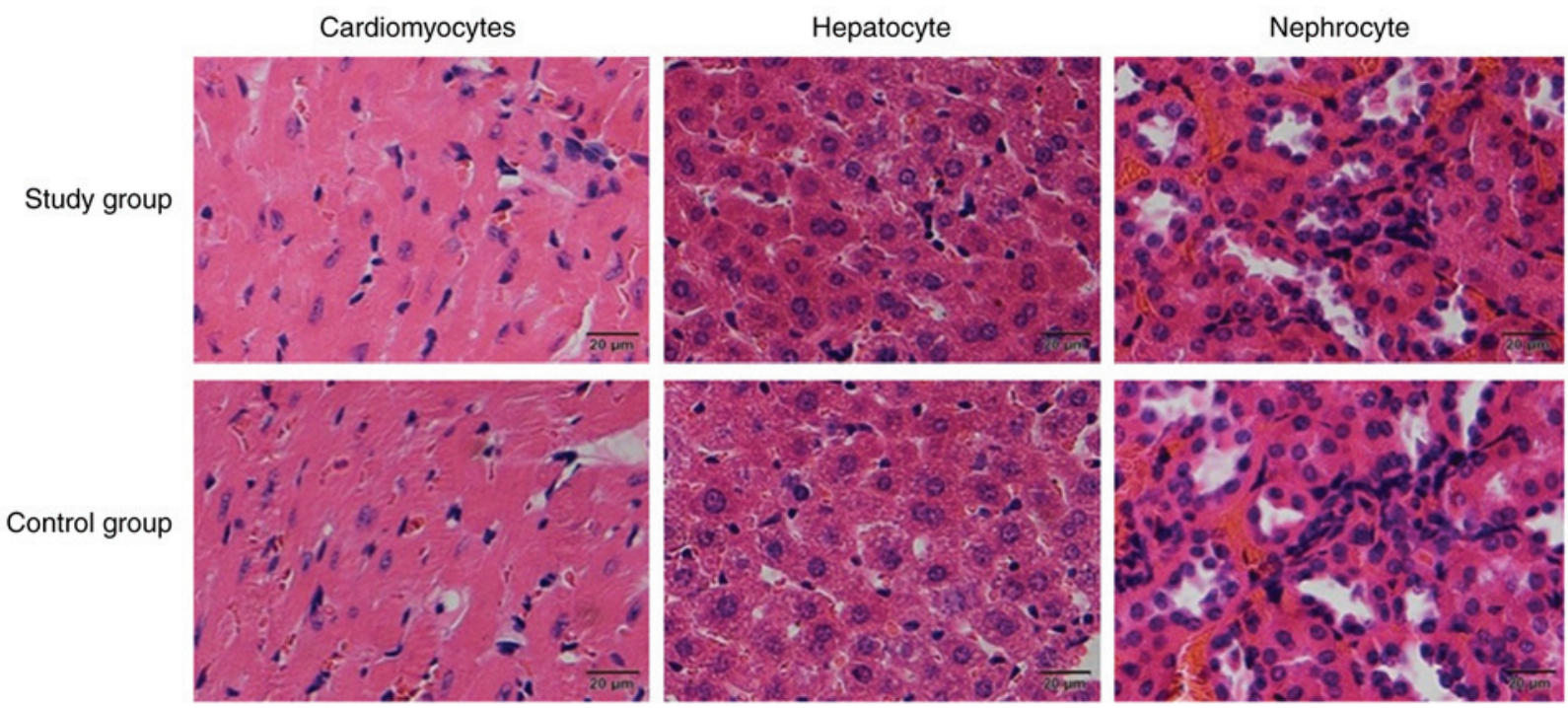

Figure 6. Hematoxylin and eosin staining of myocardial, hepatic and nephridial tissue following implantation of the porcine DM carrier in mice enterocoelia.

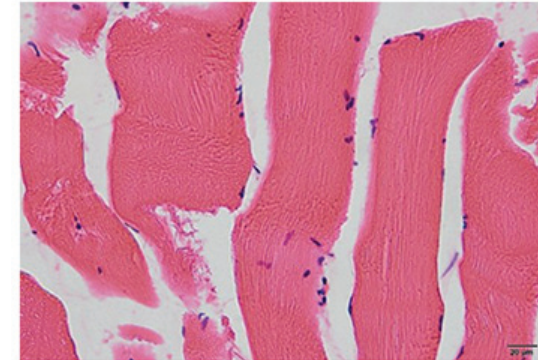

Control group

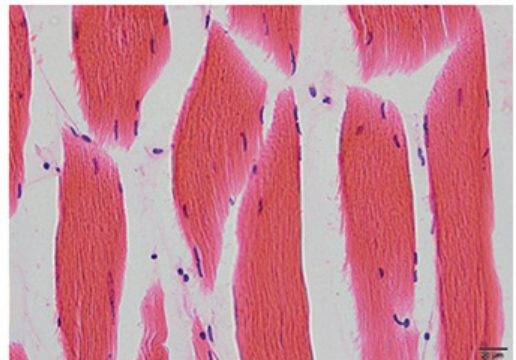

Study group

Figure 7. Hematoxylin and eosin staining of rabbit paravertebral muscle tissue following implantation of the porcine DM carrier in rabbit paravertebral muscle.

Fig. 9A and Table III). The tension in the DM-RCEC implant (experimental group) and the fresh porcine DM-endothelium complex (control) was measured, however, there was no significant difference between the experimental and control groups observed ( $\mathrm{P}>0.05$; Fig. 9B and Table IV).

\section{Discussion}

CECs form a barrier between the aqueous humour and the corneal stroma to maintain corneal clarity and thickness (8). Loss of these cells due to disease, injury or aging could result in requiring endothelial keratoplasty. However, a global shortage of donor corneas has limited the availability of endothelial keratoplasty (1). This problem can be addressed by fabricating tissue-engineered CEC implants. The CEC implants primarily include actively proliferating seed cells with a biocompatible carrier (19). The earliest method of transplanting in vitro-cultured CECs involved injecting a cell suspension into the anterior chamber of the eye. Unfortunately, the CECs adhered to the DM and to crystals, irises and angles, causing complications such as anterior chamber fibrin exudation and glaucoma (20). Over the years, scientists have searched for suitable carriers for seeding and transplanting CECs. The optimal carrier should meet the following requirements: i) The carrier must be permeable to ensure the effective exchange of
Table III. Action potential amplitudes of the implant RCECs (experimental group) and the freshly isolated RCECs (control group).

\begin{tabular}{lcc}
\hline Group & Action potential $(\mathrm{mV})$ & $\mathrm{n}$ \\
\hline Experimental group & $-81.90 \pm 2.025$ & 10 \\
Control group & $-90.30 \pm 2.406$ & 10 \\
P-value & $<0.05$ & \\
\hline
\end{tabular}

RCECs, rabbit corneal endothelial cells.

Table IV. Tension of the DM-RCEC implant (experimental group) and the fresh porcine DM-endothelium complex (control group).

\begin{tabular}{lcc}
\hline Group & Tension $(\mathrm{g})$ & $\mathrm{n}$ \\
\hline Experimental group & $20.0248 \pm 1.048$ & 10 \\
Control group & $20.5013 \pm 0.657$ & 10 \\
P-value & 0.848 & \\
\hline
\end{tabular}

DM, descemet membrane; RCECs, rabbit corneal endothelial cells. 

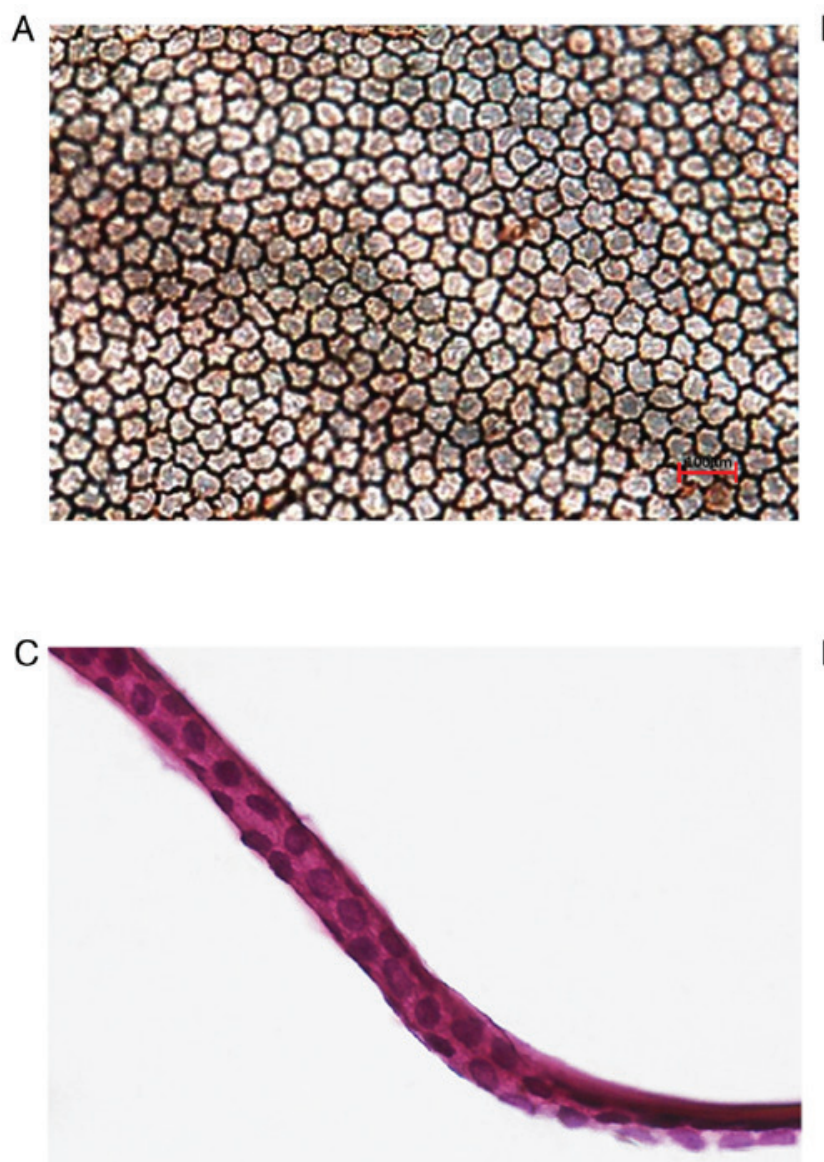

$50 \mu \mathrm{m}$

E

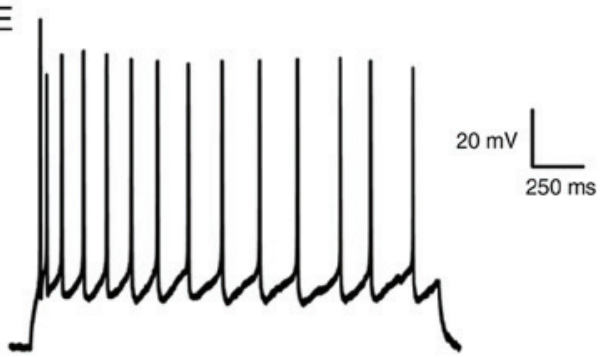

$B$

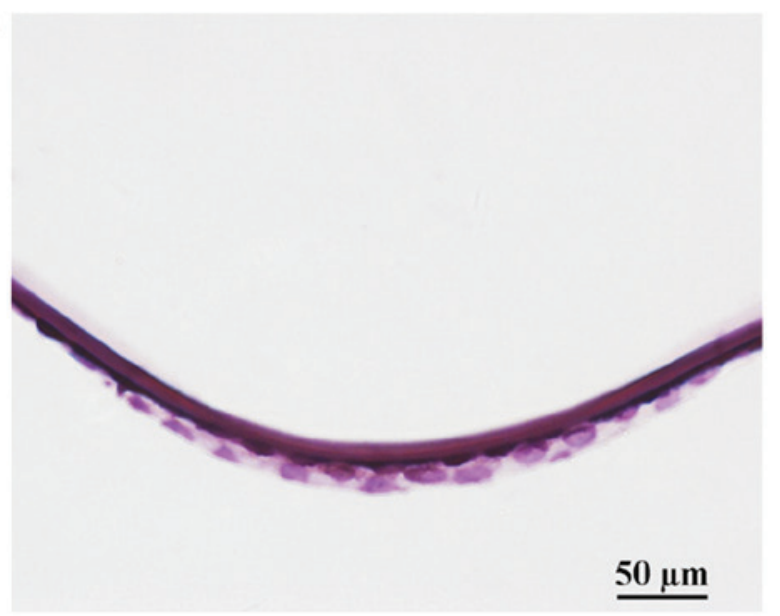

D

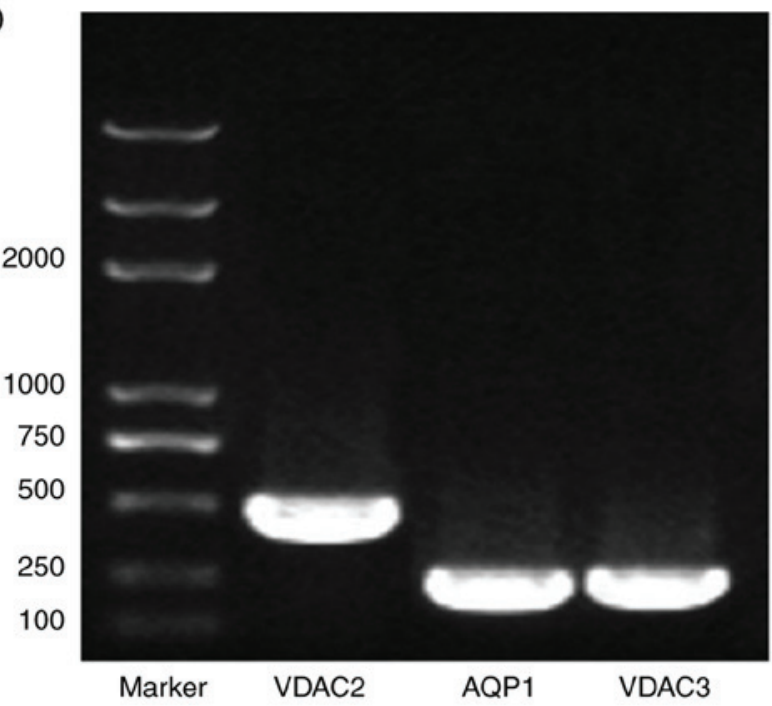

$\mathrm{F}$

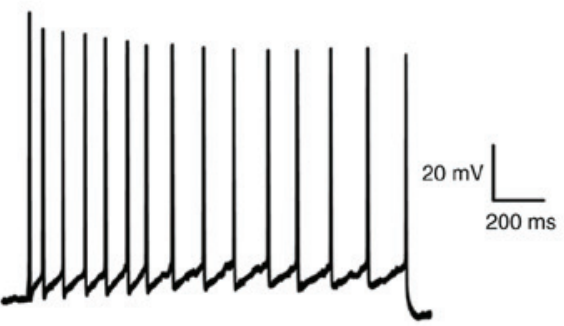

Figure 8. Confirmation of successful RCEC implantation. (A) The implanted RCECs were stained with alizarin red-trypan blue. (B) Experimental group RCECs were seeded and tightly adhered onto the porcine DM endothelium surface. (C) Control group RCECs were tightly adhered onto the DM endothelium surface of fresh RCEC-DM complexes. (D) Electrophoresis results demonstrate expression of VDAC2, VDAC3 and AQP1 in implant RCECs. The action potential amplitudes of the (E) experimental and (F) control groups. RCEC, rabbit corneal endothelial cell; AQP1, aquaporin 1.

substances between cells and the environment; ii) the carrier must be penetrable to refracting media; iii) the carrier must have sufficient tension to tolerate the transplant operation; and iv) the antigenicity of the carrier should be minimized so that the transplant is not rejected (3).

A previous study demonstrated that the peripheral cornea contains corneal endothelial precursor cells with strong renewing capacity that are suitable for transplant experiments (21). However, in vitro-cultured HCECs have reduced proliferative capacity and are subject to morphological changes (22). HCECs are affected by various factors, including endothelin and fibroblast growth factors (23-25). Y-27632, a selective ROCK inhibitor, has been shown to promote CEC adhesion and proliferation and reduce apoptosis $(26,27)$. This phenomenon may occur as Y-27632 can affect molecular compositions that regulate the cell cycle (28), although this hypothesis requires further verification. In the current study, Y-27632 was added to the cell culture medium, and the results demonstrated that Y-27632 promoted RCEC proliferation, reduced apoptosis and facilitated the cells to maintain their original morphology.

Primary cultured RCECs are easily contaminated by other cells, including corneal epithelial cells and stroma cells. $\mathrm{Na}^{+}-\mathrm{K}^{+}$-ATPase is highly expressed on CECs and is primarily distributed on both sides of the cell membrane (29). AQP1 is the only member of the AQP channel family present on CECs, and CEC transmembrane functional activity is reflected by the 

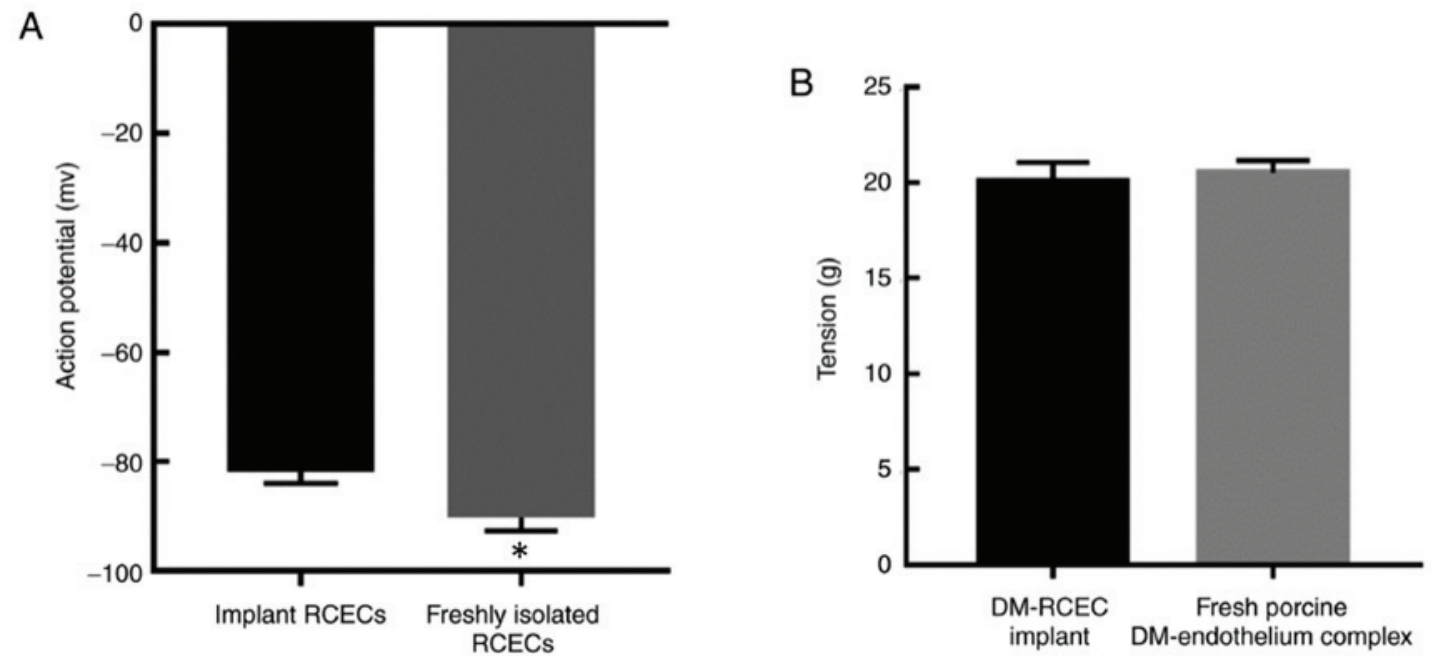

Figure 9. Cell membrane potential and DM-RCEC implant tension. (A) Action potential amplitudes of the implant RCECs (experimental group) and freshly isolated RCECs (control group). (B) Tension of the porcine DM-RCEC implant (experimental group) and fresh porcine DM endothelium complex (control group). ${ }^{*} \mathrm{P}<0.05$ vs. implant RCECs. RCEC, rabbit corneal endothelial cell; DM, Descemet membrane.

expression level of AQP1 (30). DM is primarily composed of collagen $\alpha 2$ (IV) and collagen $\alpha 1$ (VIII), which are also positively expressed on CECs (8). Keratin-12 is a known marker for corneal epithelial cells, however, it is not expressed on CECs (31). The spindle morphology of corneal stroma cells can be used to identify and isolate them from CECs (29). In the current study, morphological observations and expression of endothelial-associated markers demonstrated that the cultured cells were uncontaminated RCECs.

Previous studies have examined different carriers for RCEC implant fabrication; however, these approaches are associated with certain limitations, which include unsatisfactory biocompatibility and unsuitable differences in structure and biochemical composition (32). McCulley et al (33) reported the use of a gelatine film as a carrier to transplant in vitro-cultured CECs, however, the adhesive elements demonstrated toxicity, and a liquid gap formed between the implant bed and the gelatine film that induced fibroblast ingrowth. Liang et al (34) used a hydrogel as a carrier, however, this material caused corneal allograft rejection and changed the postoperative corneal curvature. In 2004, Ishino et al (35) used an amniotic membrane to seed CECs, resulting in high-density CECs with normal morphology and tight junctions. However, the amniotic membrane is a thick and opaque membrane, which did not conform to the cornea's physiological characteristics. In the present study, the use of a porcine DM as a carrier demonstrated several advantages. Porcine DM is widely available and easy to obtain. In addition, porcine DM has a similar thickness and shape to human DM as well as being transparent and permeable (36). DM is composed of the same collagens that are secreted by CECs (37). DM has no cellular components and is therefore characterised by a low antigenicity (36).

To use a heterogeneous DM as a carrier, the indigenous CECs must first be removed. During this process, the structure and functional proteins of the extracellular matrix should be carefully preserved (38). Gospodarowicz and Greenburg (39) revealed that chemical processes can be used to remove indigenous CECs, however, the chemicals also destroyed the fibronectin on the DM surface (40). A mechanical erasing process was previously identified as an appropriate method to prepare a carrier prior to the seeding of cultured CECs (41). In the current study, the carrier was preserved in glycerol after liquid nitrogen storage. This method was used to reduce antigenicity. The H\&E staining revealed that the carrier had no immunogenicity and potentially only a small chance of rejection.

To confirm whether an implant can be used for endothelial transplantation, cultured CECs must have an adequate density and possess normal electrophysiological activity. In addition, an implant must have sufficient tension to withstand the transplant operation. In the current study, alizarin red-trypan blue staining demonstrated that implanted RCECs maintained the structure and morphology associated with RCECs. VDAC2 and VDAC3 are members of the mitochondrial porin family, which are located in the mitochondrial outer membrane (42). CECs contain a large number of mitochondria and express high levels of VDAC2 and VDAC3 (43). In the current study, the expression levels of AQP1, VDAC2 and VDAC 3 of the implanted RCECs were consistent with that of in vivo RCECs.

Neher and Akamann (44) established the patch clamp technique to detect cellular membrane potential. In the current study, the membrane potential of the in vitro-cultured RCECs was recorded using the whole-cell mode patch clamp technique. Action potentials were observed in the implanted RCECs, however the amplitude was significantly lower than that of freshly isolated RCECs. Furthermore, the tension of implanted RCECs was examined using a homemade tension detector. The results demonstrated that the porcine DM-RCEC complex possessed sufficient tension to tolerate a transplant operation.

To the best of our knowledge, this is the first report describing the fabrication of a porcine DM-RCEC complex, as well as the use of a novel tension detection method. The long-term effects associated with the use of porcine DM-RCEC complex transplants in vivo remains unknown. Therefore, further animal experiments are required to observe the long-term effects. In addition, future work is required to improved cell culture techniques and cell sourcing, which may increase seed cell viability. 


\section{Acknowledgements}

Not applicable.

\section{Funding}

The present study was supported by grants from the Shanghai Scientific and Technical Innovation Plan 2016, experimental animal research project (grant no. 16140900900) and the Municipal Human Resources Development Program for Outstanding Leaders in Medical Disciplines in Shanghai (grant no. 2017BR060).

\section{Availability of data and materials}

The datasets used and/or analyzed during the present study are available from the corresponding author on reasonable request.

\section{Authors' contributions}

CL collected and analyzed the data, and prepared the manuscript. ST, GN and JZ performed the animal experiments and obtained tissue samples. $\mathrm{XH}$ and $\mathrm{YZ}$ performed laboratory experiments and collected the data. YB analyzed the results and revised the manuscript. All authors read and approved the final manuscript.

\section{Ethics approval and consent to participate}

This study was approved by the Ethics Committee of Tongji University School of Medicine (Shanghai, China).

\section{Patient consent for publication}

Not applicable.

\section{Competing interest}

The authors declared that they have no competing interests.

\section{References}

1. Melles GR, Ong TS, Ververs B and van der Wees J: Descemet membrane endothelial keratoplasty (DMEK). Cornea 25 987-990, 2006

2. Gospodarowicz D, Greenburg G and Alvarado J: Transplantation of cultured bovine corneal endothelial cells to species with nonregenerative endothelium. The cat as an experimental model. Arch Ophthalmol 97: 2163-2169, 1979.

3. Niu G, Choi JS, Wang Z, Skardal A, Giegengack M and Soker S: Heparin-modified gelatin scaffolds for human corneal endothelial cell transplantation. Biomaterials 35: 4005-4014, 2014.

4. Fan T, Zhao J, Ma X, Xu X, Zhao W and Xu B: Establishment of a continuous untransfected human corneal endothelial cell line and its biocompatibility to denuded amniotic membrane. Mol Vis 17: 469-480, 2011.

5. Watanabe R, Hayashi R, Kimura Y, Tanaka Y, Kageyama T, Hara S, Tabata Y and Nishida K: A novel gelatin hydrogel carrier sheet for corneal endothelial transplantation. Tissue Eng Part A 17: 2213-2219, 2011.

6. Honda N, Mimura T, Usui T and Amano S: Descemet stripping automated endothelial keratoplasty using cultured corneal endothelial cells in a rabbit model. Arch Ophthalmol 127: 1321-1326, 2009.

7. Johnston MC, Noden DM, Hazelton RD, Coulombre JL and Coulombre AJ: Origins of avian ocular and periocular tissues. Exp Eye Res 29: 27-43, 1979.
8. Edelhauser HF: The balance between corneal transparency and edema: The proctor lecture. Invest Ophthalmol Vis Sci 47: 1754-1767, 2006

9. Joyce NC, Meklir B, Joyce SJ and Zieske JD: Cell cycle protein expression and proliferative status in human corneal cells. Invest Ophthalmol Vis Sci 37: 645-655, 1996.

10. Mimura T, Yamagami S and Amano S: Corneal endothelial regeneration and tissue engineering. Prog Retin Eye Res 35: 1-17, 2013.

11. Peh GS, Beuerman RW, Colman A, Tan DT and Mehta1 JS: Human corneal endothelial cell expansion for corneal endothelium transplantation: An overview. Transplantation 91: 811-819, 2011.

12. Nakagawa H, Yoshioka K, Miyahara E, Fukushima Y, Tamura M and Itoh K: Intrathecal Administration of Y-27632, a specific Rho-associated kinase inhibitor, for rat neoplastic meningitis. Mol Cancer Res 3: 425-433, 2005.

13. Feng ZH, Zhang XH, Zhao JQ and Ma JZ: Involvement of Rho-associated coiled-coil kinase signaling inhibition in TGF- $31 /$ Smad2, 3 signal transduction in vitro. Int J Ophthalmol 10: 1805-1811, 2017.

14. Okumura N, Inoue R, Okazaki Y, Nakano S, Nakagawa $\mathrm{H}$, Kinoshita $\mathrm{S}$ and Koizumi N: Effect of the Rho kinase inhibitor Y-27632 on corneal endothelial wound healing. Invest Ophthalmol Vis Sci 56: 6067-6074, 2015.

15. Zhang J, Cai H, Sun L, Zhan P, Chen M, Zhang F, Ran Y and Wan J: LGR5, a novel functional glioma stem cell marker, promotes EMT by activating the $\mathrm{Wnt} / \beta$-catenin pathway and predicts poor survival of glioma patients. J Exp Clin Cancer Res 37: 225-231, 2018

16. Livak KJ and Schmittgen TD: Analysis of relative gene expression data using real-time quantitative PCR and the 2(-Delta Delta C(T)) method. Methods 25: 402-408, 2001.

17. Serafini S, Santos MM, Aoun Tannuri AC, Zerbini MCN, de Mendonça Coelho MC, de Oliveira Gonçalves J and Tannuri U: Is hematoxylin-eosin staining in rectal mucosal and submucosal biopsies still useful for the diagnosis of Hirschsprung disease? Diagn Pathol 12: 84, 2017.

18. Leyrer-Jackson JM, Olive MF and Gipson CD: Whole-cell patch-clamp electrophysiology to study ionotropic glutamatergic receptors and their roles in addiction. Methods Mol Biol 1941: 107-135, 2019.

19. Esipov RS, Beřrakhova KA, Chupova LA, Likhvantseva VK, Stepanova EV and Miroshnikov AI: Recombinant fragment of pigment epithelium-derived factor (44-77) prevents pathological corneal neovascularization. Bioorg Khim 38: 78-85, 2012 (In Russian).

20. Singh N, Higgins E, Amin S, Jani P, Richter E, Patel A, Kaur R, Wang J, Ambati J, Dong Z and Ambati BK: Unique homologous siRNA blocks hypoxia-induced VEGF upregulation in human corneal cells and inhibits and regresses murine corneal neovascularization. Cornea 26: 65-72, 2007.

21. Mimura T, Yamagami S, Yokoo S, Araie M and Amano S: Comparison of rabbit corneal endothelial cell precursors in the central and peripheral cornea. Invest Ophthalmol Vis Sci 46: 3645-3648, 2005.

22. Okumura N, Kay EP, Nakahara M, Hamuro J, Kinoshita S and Koizumi N: Inhibition of TGF- $\beta$ signaling enables human corneal endothelial cell expansion in vitro for use in regenerative medicine. PLoS One 8: e58000, 2013.

23. Rieck P, Oliver L, Engelmann K, Fuhrmann G, Hartmann C and Courtois Y: The role of exogenous/endogenous basic fibroblast growth factor (FGF2) and transforming growth factor beta (TGF beta-1) on human corneal endothelial cells proliferation in vitro. Exp Cell Res 220: 36-46, 1995.

24. Soldano S, Paolino S, Pizzorni C, Trombetta AC, Montagna P, Brizzolara R, Corallo C, Giordano N, Sulli A and Cutolo M: Dual endothelin receptor antagonists contrast the effects induced by endothelin-1 on cultured human microvascular endothelial cells. Clin Exp Rheumatol 35: 484-493, 2017.

25. Hsieh P and Baum J: Effects of fibroblastic and endothelial extracellular matrices on corneal endothelial cells. Invest Ophthalmol Vis Sci 26: 457-463, 1985.

26. Okumura N, Koizumi N, Ueno M, Sakamoto Y, Takahashi H, Hirata K, Torii R, Hamuro J and Kinoshita S: Enhancement of corneal endothelium wound healing by Rho-associated kinase (ROCK) inhibitor eye drops. Br J Ophthalmol 95: 1006-1009, 2011.

27. Bi YL, Zhou Q, Du F, Wu MF, Xu GT and Sui GQ: Regulation of functional corneal endothelial cells isolated from sphere colonies by Rho-associated protein kinase inhibitor. Exp Ther Med 5: 433-437, 2013. 
28. Joyce NC and Harris DL: Decreasing expression of the G1-phase inhibitors, p21Cip1 and p16INK4a, promotes division of corneal endothelial cells from older donors. Mol Vis 16: 897-906, 2010.

29. Ding V, Chin A, Peh G, Mehta JS and Choo A: Generation of novel monoclonal antibodies for the enrichment and characterization of human corneal endothelial cells (hCENC) necessary for the treatment of corneal endothelial blindness. MAbs 6: 1439-1452, 2014

30. Song Q, Yuan S, An Q, Chen Y, Mao FF, Liu Y, Liu Q and Fan G: Directed differentiation of human embryonic stem cells to corneal endothelial cell-like cells: A transcriptomic analysis. Exp Eye Res 151: 107-114, 2016.

31. Wu RL, Zhu G, Galvin S, Xu C, Haseba T, Chaloin-Dufau D, Dhouailly D, WeiZG,LavkerRM,Kao WY, et al:Lineage-specific and differentiation-dependent expression of K12 keratin in rabbit corneal/limbal epithelial cells: cDNA cloning and northern blot analysis. Differentiation 55: 137-144, 1994.

32. Ahearne M, Wilson SL, Liu KK, Rauz S, El Haj AJ and Yang Y: Influence of cell and collagen concentration on the cell-matrix mechanical relationship in a corneal stroma wound healing model. Exp Eye Res 91: 584-591, 2010.

33. McCulley JP, Maurice DM and Schwartz BD: Corneal endothelial transplantation. Ophthalmology 87: 194-201, 1980.

34. Liang Y, Liu W, Han B, Yang C, Ma Q, Song F and Bi Q: An in situ formed biodegradable hydrogel for reconstruction of the corneal endothelium. Colloids Surf B Biointerfaces 82: 1-7, 2011.

35. Ishino Y, Sano Y, Nakamura T, Connon CJ, Rigby H, Fullwood NJ and Kinoshita S: Amniotic membrane as a carrier for cultivated human corneal endothelial cells transplantation. Invest Ophthalmol Vis Sci 45: 800-806, 2004.

36. Diao YM and Hong J: Feasibility and safety of porcine Descemet's membrane as a carrier for generating tissue-engineered corneal endothelium. Mol Med Rep 12: 1929-1934, 2015.
37. Choi HJ, Kim MK, Lee HJ, Ko JH, Jeong SH, Lee JI, Oh BC, Kang HJ and Wee WR: Efficacy of pig-to-rhesus lamella corneal xenotransplantation. Invest Ophthalmol Vis Sci 52: 6643-6650, 2011.

38. Murphy SV and Atala A: Organ engineering-combining stem cells, biomaterials, and bioreactors to produce bioengineered organs for transplantation. Bioessays 35: 163-172, 2013.

39. Gospodarowicz D and Greenburg G: The coating of bovine and rabbit corneas denuded of their endothelium with bovine corneal endothelial cells. Exp Eye Res 28: 249-265, 1979.

40. Chen KH, Azar D and Joyce NC: Transplantation of adult human corneal endothelium ex vivo: A morphologic study. Cornea 20: 731-737, 2001.

41. Jumblatt MM, Maurice DM and McCulley JP: Transplantation of tissue-cultured corneal endothelium. Invest Ophthalmol Vis Sci 17: 1135-1141, 1978 .

42. Thinnes FP: Neuroendocrine differentiation of LNCaP cells suggests: VDAC in the cell membrane is involved in the extrinsic apoptotic pathway. Mol Genet Metab 97: 241-243, 2009.

43. McIntosh Ambrose W, Salahuddin A, So S, Ng S, Ponce Márquez S Takezawa T, Schein O and Elisseeff J: Collagen Vitrigel membranes for the in vitro reconstruction of separate corneal epithelial, stromal, and endothelial cell layers. J Biomed Mater Res B Appl Biomater 90: 818-831, 2009.

44. Neher E and Sakmann B: Single channel currents recorded from membrane of denervated frog muscle fibers. Nature 260: 799-802, 1976.

This work is licensed under a Creative Commons Attribution-NonCommercial-NoDerivatives 4.0 International (CC BY-NC-ND 4.0) License. 